\title{
Robust Gaussian Process Regression Based on Iterative Trimming
}

\author{
Zhao-Zhou Lid ${ }^{\mathrm{a}, 1, *}$, Lu Li $^{\mathrm{b}, \mathrm{c}, 2}$, Zhengyi Shao ${ }^{\mathrm{b}, \mathrm{d}}$ \\ ${ }^{a}$ Department of Astronomy, School of Physics and Astronomy, Shanghai Jiao Tong University, 955 Jianchuan Road, Shanghai 200240, China \\ ${ }^{b}$ Key Laboratory for Research in Galaxies and Cosmology, Shanghai Astronomical Observatory, Chinese Academy of Sciences, 80 Nandan Road, Shanghai \\ 200030, China \\ ${ }^{c}$ University of the Chinese Academy of Sciences, No.19A Yuquan Road, Beijing 100049, China \\ ${ }^{d}$ Key Lab for Astrophysics, Shanghai 200234, China
}

\begin{abstract}
The Gaussian process (GP) regression can be severely biased when the data are contaminated by outliers. This paper presents a new robust GP regression algorithm that iteratively trims the most extreme data points. While the new algorithm retains the attractive properties of the standard GP as a nonparametric and flexible regression method, it can greatly improve the model accuracy for contaminated data even in the presence of extreme or abundant outliers. It is also easier to implement compared with previous robust GP variants that rely on approximate inference. Applied to a wide range of experiments with different contamination levels, the proposed method significantly outperforms the standard GP and the popular robust GP variant with the Student- $t$ likelihood in most test cases. In addition, as a practical example in the astrophysical study, we show that this method can precisely determine the main-sequence ridge line in the color-magnitude diagram of star clusters.
\end{abstract}

Keywords: Gaussian process, robust regression, outlier detection, ridge line, star clusters

\section{Highlights}

- Nonparametric, flexible, and robust regression based on Gaussian process.

- Outperforming the robust Gaussian process with Student- $t$ likelihood significantly in many test cases.

- Easy to implement and computationally tractable.

- Practical example in the astrophysical study.

\section{Introduction}

There has been increasing interest in the Gaussian process (GP) regression (Rasmussen and Williams, 2005) method in both scientific research and industry applications. As a nonparametric method, GP is completely data-driven. It does not assume any explicit functional form between variables, which is particularly attractive in the big data era. Moreover, GP provides a Bayesian framework which can naturally characterize prior and posterior distributions over functions. As the basis of Bayesian optimization, GP can serve as a probabilistic surrogate model for problems that demand sample efficiency. For example, the cosmological emulators based on GP (e.g., Heitmann et al. 2009; McClintock et al. 2019) can make precise predictions in large parameter space using merely a finite set of numerical simulations. This technique can save a substantial investment of time and resources because each simulation is highly computation demanding.

\footnotetext{
*Corresponding author

${ }^{1}$ lizz.astro@gmail.com

2 lilu@shao.ac.cn
}

GP can naturally handle the noises that are assumed to follow normal distributions. However, predictions under this assumption are highly susceptible to the presence of extreme observations in data, the so-called outliers. Outliers are usually generated by mechanisms different from the main sample, for example, failure in measurement or calculation (e.g., broken sensor), external factors (e.g., cosmic ray in astronomical images), and insufficient explanatory power of the model (e.g., binary stars for single stellar population models). Their presence can make the estimate deviate substantially from the expected value, as illustrated in Fig. 1. Therefore, robust regression techniques become necessary in such cases (see Huber and Ronchetti 2009 and Maronna et al. 2019 for general reviews on robust statistics).

Several robust variants of GP regression have been proposed. The most popular approach is to use alternative observation models that are less sensitive to extreme values, for example, the heavy-tailed distributions such as Student- $t$ (Neal, 1997; Vanhatalo et al., 2009; Jylänki et al., 2011; Ranjan et al., 2016) or Laplace (Kuß, 2006) distribution, mixture of multiple Gaussians (Kuß, 2006; Stegle et al., 2008; Ross and Dy, 2013), and input dependent noise model (Goldberg et al., 1998; Naish-Guzman and Holden, 2007; Almosallam, 2017). However, unlike the Gaussian noise case, GPs with these models become analytically intractable, hence relying on approximate inference techniques, e.g., variational approximation and expectation propagation, which are often challenging to understand and implement. More importantly, they may still suffer from the influence of outliers to some extent, as shown in Fig. 1 for GP with Student- $t$ likelihood ( $t$-lik).

Another sensible strategy is to trim the outliers with large deviations from the GP model prediction, then rerun GP on 


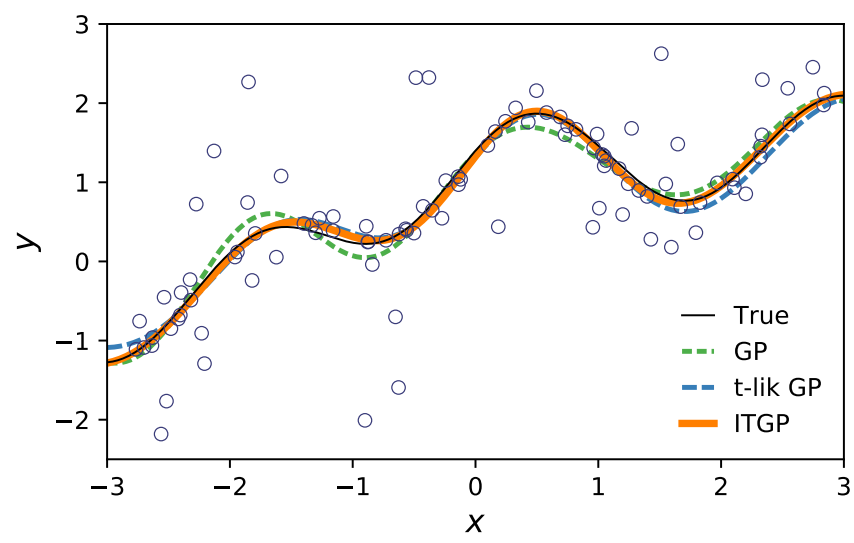

Figure 1: Illustration of the performance of three GP regression methods in the presence of outliers. $45 \%$ of the sample is corrupted with noises ten times that of the main sample (see Case "abundant" in Section 3.1 for details). The model prediction from the standard GP (green) is significantly biased, making robust GP methods necessary for better accuracy. Compared with the Student- $t$ likelihood GP (blue), the proposed ITGP (orange) shows better performance in this test case.

the purified sample. However, noting that the initial model prediction itself is already affected by outliers, the consequent residuals can not faithfully reveal the true deviations, possibly leading to severe bias. While this effect can be mitigated using iterations (e.g., Wang et al. 2017), it is a nontrivial task to figure out a general procedure that achieves both high robustness and high statistical efficiency with the minimal possible computation cost.

This work proposes a new robust regression method based on the standard GP and iterative trimming (denoted as ITGP), which is easy to implement and computationally tractable. As shown in this paper, while the new method retains the GP's attractive properties as a nonparametric and flexible regression method, it shows robust performance in the presence of outliers and outperforms the popular $t$-lik GP significantly in many cases.

The rest of the paper is organized as follows. In Section 2, we first provide some preliminaries of the GP regression, then present the proposed ITGP algorithm and corresponding practical guidance. We compare the performance of ITGP and other methods for a variety of test cases in Section 3 and summarize in Section 4.

Alongside this paper, we have released our open-source implementation of ITGP online (https : //github. com/syrte/ robustgp/), which is written in PyтноN based on the public GP package GPy.

\section{Robust Gaussian Process Regression}

\subsection{Basis of Gaussian process}

We consider a regression problem

$$
y=f(\boldsymbol{x})+\epsilon,
$$

where the observable (aka response or target) $y$ is the summation of an underlying model, $f(\boldsymbol{x}): \mathbb{R}^{d} \rightarrow \mathbb{R}$, and an observation noise $\epsilon$. The object is to infer the latent function $f(\boldsymbol{x})$ from a dataset $\mathcal{D}=\left\{\boldsymbol{x}_{i}, y_{i}\right\}_{i=1}^{n}$, where $n$ is the sample size.

The Gaussian process (GP) provides a flexible prior on a family of functions. If the underlying model $f$ is a realization of a GP with a mean function $m(\boldsymbol{x})$ and a kernel function $k\left(\boldsymbol{x}, \boldsymbol{x}^{\prime}\right)$, then the function values at arbitrary subset of locations, $f=$ $\left\{f\left(\boldsymbol{x}_{i}\right)\right\}_{i=1}^{n}$, follow a multivariate Gaussian distribution,

$$
p(\boldsymbol{f})=\mathcal{N}(\boldsymbol{f} \mid \boldsymbol{\mu}, \boldsymbol{\Sigma}) .
$$

Here the mean vector and covariance matrix are determined by $\boldsymbol{\mu}_{i}=m\left(\boldsymbol{x}_{i}\right)$ and $\boldsymbol{\Sigma}_{i, j}=k\left(\boldsymbol{x}_{i}, \boldsymbol{x}_{j}\right)$ respectively. Conventionally, people use a zero mean function and an analytical kernel function that is controlled by a set of hyper-parameters $\Theta$ (see Duvenaud 2014 for discussion on choice of kernel).

The standard GP assumes that the observation noise $\epsilon$ also follows a Gaussian distribution, then $\boldsymbol{y}=\left\{y_{i}\right\}_{i=1}^{n}$ is still Gaussian and can be reinterpreted by adding a white noise term in the kernel $k$, see Equation (6) for an example. It is particularly convenient because the inference is analytically tractable.

Given a training sample, $\mathcal{D}=\left\{\boldsymbol{x}_{i}, y_{i}\right\}_{i=1}^{n}$, one can first infer the optimal hyper-parameters $\Theta$ for the kernel by maximizing the likelihood $p\left(\boldsymbol{y} \mid\left\{\boldsymbol{x}_{i}\right\}, \Theta\right)$, then use the conditional distribution to derive the posterior prediction $p\left(f_{*} \mid \mathcal{D}, \Theta\right)$ at any new point $\boldsymbol{x}_{*}$, including the mean $\hat{f}_{*}=\mathbb{E}\left[f_{*}\right]$ and the variance $\sigma_{*}^{2}=\operatorname{var}\left[f_{*}\right]$. See Rasmussen and Williams (2005) for details.

As mentioned in the introduction, inferences with GPs are susceptible to outliers, which return extremely low likelihood under the Gaussian distribution. To alleviate their influence, one can keep $f$ as a realization of the GP but use alternative observation models for $\epsilon$, e.g., Student- $t$ distribution, whose long tail can lower the significance of the points with large deviation. However, note that $p(y)$, as the convolution of a Gaussian and a Student- $t$ distribution, does not have an explicit form. Therefore, the likelihood and the conditional posterior are no longer analytically tractable hence requiring approximate inference techniques, which are generally more challenging in both implementation and computation.

In the following, we present our new method ITGP, which aims to sustain the simplicity of the standard GP and strengthen the robustness simultaneously.

\subsection{Robust Gaussian process with iterative trimming}

The main idea of ITGP is to iteratively trim a proportion of the points with the largest absolute residuals, so that the remaining sample can better describe the bulk pattern of the data. This method is summarized in Algorithm 1 and illustrated in Fig. 2. Training an ITGP model consists of three stages: shrinking, concentrating, and reweighting.

- Shrinking and concentrating stages (lines 2-15). The procedure is as follows.

1. First train the standard GP with the full sample $\left\{\boldsymbol{x}_{i}, y_{i}\right\}_{i=1}^{n}$, predict the mean $\hat{f}_{i}$ and variance $\sigma_{i}^{2}=\operatorname{var}\left[\hat{y}_{i}\right]$ for each point, and calculate corresponding normalized residual, $r_{i}^{\prime}=\left|y_{i}-\hat{f}_{i}\right| / \sigma_{i}$.

2. Retrain the GP using the $\lceil\alpha n\rceil$ points with the smallest residuals $r^{\prime}$ and update the predictions $\left\{\hat{f}_{i}, \sigma_{i}, r_{i}^{\prime}\right\}_{i=1}^{n}$. 


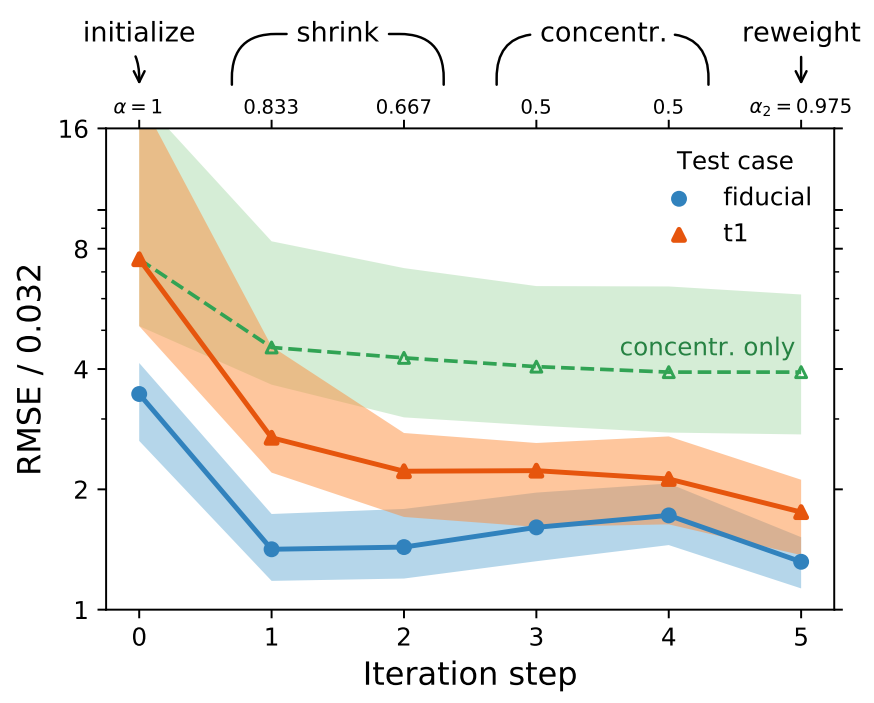

Figure 2: Illustration of the iterative trimming with two test cases. The solid curves show the median of the root-mean-square error (RMSE, scaled by 0.032 for clarity) of the prediction as a function of iteration steps, and the shaded bands show the interquartiles. Each case contains 50 datasets, and each dataset contains 100 data points (see Section 3.1). For each dataset and at each step, a proportion (characterized by $\alpha$ ) of points with the largest residuals is removed, then a GP is retrained with the trimmed sample. The shrinking stage can prevent premature convergence and improve model accuracy, while the reweighting step can increase the statistical efficiency. For comparison, the dashed curve shows results for Case " $t_{1}$ " obtained by concentrating without shrinking and reweighting stages.

3. Repeat Step 2 for $n_{\mathrm{sh}}+n_{\mathrm{cc}}$ times. Let the preserving fraction $\alpha$ shrink from 1 to $\alpha_{1}$ gradually in the first $n_{\mathrm{sh}}$ iterations (shrinking stage) and remain constant for the next $n_{\mathrm{cc}}$ iterations (concentrating stage). Here $\alpha_{1}, n_{\mathrm{sh}}$, and $n_{\mathrm{cc}}$ are ITGP parameters.

Note that the $\lceil\alpha n\rceil$ points are always selected from the full sample so that a point inappropriately discarded in an earlier iteration could be brought back later. For the first few iterations, the predicted mean might deviate from the underlying function substantially due to the influential outliers. However, as shown in Fig. 2, the deviation decreases fast because we always dispose of the outermost points in each iteration step. We also find that a non-zero $n_{\text {sh }}$ can effectively prevent premature convergence and significantly improve the model accuracy.

It is known that the variance of a $(1-\alpha)$-trimmed sample underestimates the actual variance of the underlying sample by a consistency factor, $c$ (Croux and Haesbroeck 1999, see also Pison et al. 2002 for the finite-sample correction). Under normality,

$$
c=\alpha / F_{\chi_{3}^{2}}\left(\chi_{1, \alpha}^{2}\right),
$$

where $F_{\chi_{3}^{2}}$ is the cumulative distribution function of the $\chi_{k=3}^{2}$ distribution (3-DoF $\chi^{2}$ ) and $\chi_{1, \alpha}^{2}$ is the $\alpha$-quantile of the $\chi_{k=1}^{2}$ distribution. As reference, $c=2.65^{2}, 1.65^{2}$ for $\alpha=0.5,0.75$ respectively. Therefore, the corrected normalized residual writes

$$
r_{i}=\left|y-\hat{f_{i}}\right| /\left(\sigma_{i} \sqrt{c}\right),
$$

where $\sigma_{i}^{2}$ is the variance predicted by the GP trained with the trimmed sample.

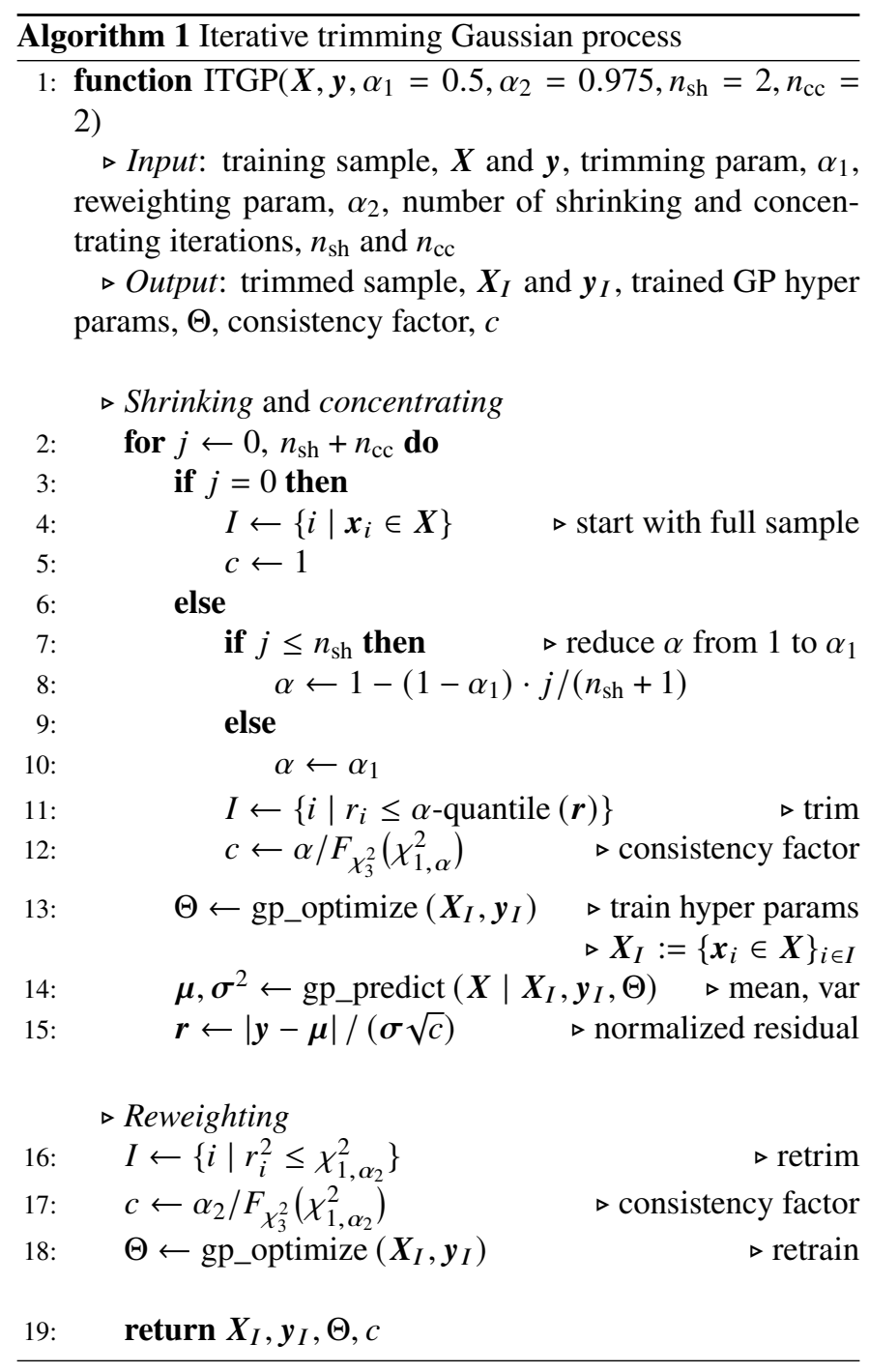

- Reweighting stage (lines 16-18). To increase the statistical efficiency, one can further apply a one-step reweighting. A simple yet effective choice (e.g., Rousseeuw and Leroy, 1987) is to remove the data points with $r_{i}^{2}>\chi_{1, \alpha_{2}}^{2}$ and retrain the GP with the remaining sample. Under normality, this amounts to discarding a proportion of about $1-\alpha_{2}$ of the points with the largest absolute residuals. It is customary to take $\alpha_{2}=0.95$ or 0.975 (corresponding to $\chi_{1}^{2}=1.96^{2}, 2.24^{2}$ and $c=1.15^{2}$, $\left.1.08^{2}\right)$.

Finally, once the ITGP is finalized, one can then identify outliers by the corrected normalized residuals $r_{i}$, e.g., label the data points with $r_{i}>3$ as $3 \sigma$ outliers (under normality). Besides, the quantile-quantile (Q-Q) plot can also be helpful diagnostics.

\subsection{Practical guidance}

To the best of our knowledge, we recommend the following ITGP parameters for general problems: $\alpha_{1}=0.5, n_{\mathrm{sh}}=2$, $n_{\mathrm{cc}}=2$, and $\alpha_{2}=0.975$.

We find the above values work remarkably well for all test cases presented in this work. According to systematic experi- 
ments in the Appendix A, further tuning can bring slight improvements in particular cases, but often at the price of higher computation cost or possibly worse performance for general problems. Hence, our recommendation appears as a good compromise among robustness, efficiency, and computation cost.

It is worth emphasizing that one should not use any method blindly in practice. Although ITGP shows general robustness against abundant and extreme outliers, it is always good practice to clean the input data beforehand, use prior information when available, and check if the model provides a reasonable summary of the bulk pattern in the data. In particular, as a flexible nonlinear regression method, ITGP could be biased by influential points near the boundary of the data coverage when the local signal-to-noise ratio is low, see Section 3.1 and Fig. 5 for example. Moreover, the first few iterations of ITGP inevitably suffer from masking and swamping effects, which might break down the whole iteration procedure in some rare cases. Our experience suggests that taking one or several of the following measures can effectively prevent catastrophic failures (e.g., dramatic oscillation in prediction) in difficult cases: removing obvious outliers by truncating the data, setting reasonable bounds (even loose ones) for GP hyperparameters (i.e., the scale length and variance of kernels), optimizing hyperparameters with a cold start (rather than values from the last iteration), and using a larger $n_{\text {sh }}$ (e.g., 5 or 10 rather than 2).

\subsection{Speed}

Given the $O\left(n^{3}\right)$ complexity of GP, naively, we expect the training time of ITGP with $n_{\mathrm{sh}}=2$ and $n_{\mathrm{cc}}=2$ to be about three times longer than the standard GP. It is a lower limit, considering the additional computations for making prediction in each iteration. According to the numerical experiments based on GPY in Section 3, the time cost of ITGP is typically 3.5 to 5 times that of the standard GP.

Obviously, the absolute time cost depends on the implementation. People have proposed many fast and scalable GP implementations (see Liu et al. 2020 for a recent review), including exact solutions with fast and parallel linear algebra algorithms (e.g., GEORGE, Ambikasaran et al. 2015; CELERITE, ForemanMackey et al. 2017) and sparse approximations (e.g., the Sparse Variational GP, Hensman et al., 2013). Given that ITGP is completely based on the standard GP, these techniques can be painlessly deployed to the ITGP for large datasets.

\subsection{Connection to LTS and other robust estimators}

There is a clear relevance between the proposed ITGP and the Least Trimmed Squares (LTS) estimators (Rousseeuw, 1984), a popular robust (linear) regression method with a high breakdown value. LTS looks for a subset of given size with the smallest mean squared residual from all possible combinations, making itself a hard combinatorial problem. Rousseeuw and Driessen (2006) proposed an efficient approximation algorithm (Fast-LTS), which contains a similar concentrating procedure (the so-called C-step). In a sense, ITGP can be seen as an approximate local solution of LTS. The concept of the global LTS solution seems attractive; however, it is nontrivial to properly define and efficiently solve it in the framework of GP regressions. For example, the flexibility of GP even allows a solution to pass through every data point. Therefore, the GP posterior probability might be a more appropriate target than the residuals. We leave such exploration to future work.

The raw LTS estimator is long known for this low efficiency. Besides the simple yet effective one-step reweighting adopted in this work, there exist other robust estimators with high efficiency in the context of robust statistics (see Yu and Yao, 2017; Maronna et al., 2019), e.g., M-estimators (Huber, 1964), MM-estimators (Yohai, 1987), and REWLSE (Gervini and Yohai, 2002). It is probably worth incorporating such techniques into GP methods as well. For example, Ramirez-Padron et al. (2021) recently proposed a robust weighted GP algorithm based on M-estimators, which shows performance comparable to and sometimes better than $t$-lik GP.

It is also possible to combine different techniques, e.g., trimming based on preceding outlier detection via clustering algorithm (Wang et al. 2017) or $t$-lik GP regression (Martinez-Cantin et al., 2018). Similarly, some advanced robust estimators like MM-estimators and REWLSE require an initial robust estimate as input, where ITGP can be a good choice.

\section{Experiments with Synthetic Datasets}

In this section, we conduct a wide range of numerical experiments to compare the performance of the proposed ITGP, the standard GP, and the robust GP variant with Student- $t$ likelihood ( $t$-lik). The $t$-lik GP has been compared with many other robust GP variants and shows broadly similar performance (e.g., Kuß 2006; Ranjan et al. 2016; Ramirez-Padron et al. 2021), hence serving as a good representative of existing methods.

The proposed ITGP is implemented in Python based on the public package GPy, and the remaining two methods are performed with GPY directly. The hyperparameters are determined by the maximum a posterior (MAP) estimation with the LBFGS-B algorithm. ${ }^{3}$ We adopt the following configurations for the three methods respectively.

- GP: the standard Gaussian process with Gaussian noise model. For reference, we also show the GP prediction (labeled as "Ideal") based on the purified sample with all contamination excluded. The latter presents the best possible performance in theory.

- $t$-lik GP: the Gaussian process with Student- $t$ noise model. We use the approximate posterior with the Laplace approximation, which shows performance very close to other implementations in terms of RMSE (Vanhatalo et al., 2009; Ranjan et al., 2016). Changing the degree of freedom $v$ of a Student- $t$ distribution allows its shape to vary from Gaussian to heavy-tailed.

- ITGP: the iterative trimming Gaussian process with Gaussian noise model. We adopt the recommended parame-

\footnotetext{
${ }^{3}$ The tests are performed on a computing node with 4 Intel Xeon Gold 6240 Processors $(2.6 \mathrm{GHz}, 4 \times 18$ cores in total). Array computations in GPY are automatically paralleled through NuMPY.
} 
ters for all test cases: $\alpha_{1}=0.5, n_{\mathrm{sh}}=2, n_{\mathrm{cc}}=2$, and $\alpha_{2}=0.975$.

We present tests for two series of artificial datasets. The first series of datasets was introduced in the seminal work of Neal (1997); the second was generated in a study on star clusters by Li et al. (2020). For each test case, 50 training datasets are generated and used to train the models separately. The trained models are then evaluated on a noise-free test set with $m=2000$ data points. Following Kuß (2006), to evaluate the performance, we report the root-mean-square error, $\operatorname{RMSE}=\left(\frac{1}{m} \sum_{i=1}^{m} \Delta_{i}^{2}\right)^{1 / 2}$, of the prediction residuals of each test set, $\left\{\Delta_{i}=f\left(x_{*, i}\right)-\right.$ $\left.\hat{f}\left(x_{*, i}\right)\right\}_{i=1}^{m}$. Consistent conclusions are obtained if using the mean absolute error instead. We also provide the computation time for reference. The average RMSE and time cost of each test case are summarized in Table 1 and 2.

\subsection{Neal datasets}

The Neal (1997) datasets have been widely used as benchmarks for robust regression methods (e.g., Vanhatalo et al. 2009; Ranjan et al. 2016). The underlying function $f(x)$ is given by

$$
f(x)=0.3+0.4 x+0.5 \sin (2.7 x)+\frac{1.1}{1+x^{2}} .
$$

To test the performance under different conditions, we consider the following noise models for observation, $y=f(x)+\epsilon$.

- Case 1: zero outliers,

$$
\epsilon \sim \mathcal{N}\left(0,0.1^{2}\right)
$$

- Case 2: rare outliers,

$$
\epsilon \sim 0.95 \mathcal{N}\left(0,0.1^{2}\right)+\mathbf{0 . 0 5} \mathcal{N}\left(0,1^{2}\right)
$$

- Case 3: fiducial case,

$$
\epsilon \sim 0.85 \mathcal{N}\left(0,0.1^{2}\right)+0.15 \mathcal{N}\left(0,1^{2}\right) .
$$

- Case 4: abundant outliers,

$$
\epsilon \sim 0.55 \mathcal{N}\left(0,0.1^{2}\right)+\mathbf{0 . 4 5} \mathcal{N}\left(0,1^{2}\right)
$$

- Case 5: skewed outliers,

$$
\epsilon \sim 0.85 \mathcal{N}\left(0,0.1^{2}\right)+0.15 \mathcal{N}\left(2,1^{2}\right) \text {. }
$$

- Case 6: extreme outliers,

$$
\epsilon \sim 0.85 \mathcal{N}\left(0,0.1^{2}\right)+0.15 \mathcal{N}\left(0, \mathbf{5}^{2}\right) .
$$

- Case 7: uniform outliers,

$$
y \sim 0.7 \mathcal{N}\left(f, 0.1^{2}\right)+\mathbf{0 . 3} \mathcal{U}[-3,3]
$$

- Case 8: $t_{3}$ distribution with $\operatorname{var}[\epsilon]=3 \times 0.1^{2}$,

$$
\epsilon \sim t\left(0,0.1^{2} ; v=3\right) \text {. }
$$

- Case 9: $t_{1}$ (aka Cauchy) distribution with $\operatorname{var}[\epsilon]=\infty$,

$$
\epsilon \sim t\left(0,0.1^{2} ; v=1\right) \text {. }
$$

Gaussian noise is assumed for the main samples in Cases 1-7, and a Student- $t$ noise is assumed in Cases 8 and 9. Moreover, Cases 2-6 are further contaminated by another Gaussian component with substantially larger $(\times 10$ or more $)$ scatter, while Case 7 is contaminated by uniformly distributed outliers. The difference between each case and the "fiducial" case is marked in bold. Cases 4 and 9 are particularly challenging problems for the excessive contamination fraction (45\%) and extremely distributed outliers $(\operatorname{var}[\epsilon]=\infty)$.

Each test case contains 50 training samples, and each sample contains $n=100$ data points randomly drawn from $x \in[-3,3]^{4}$

${ }^{4}$ It is slightly different from the original Neal (1997) dataset, where $x$ was sampled from a normal distribution. It is because we are not interested in the possible leverage points in $x$ in this work.

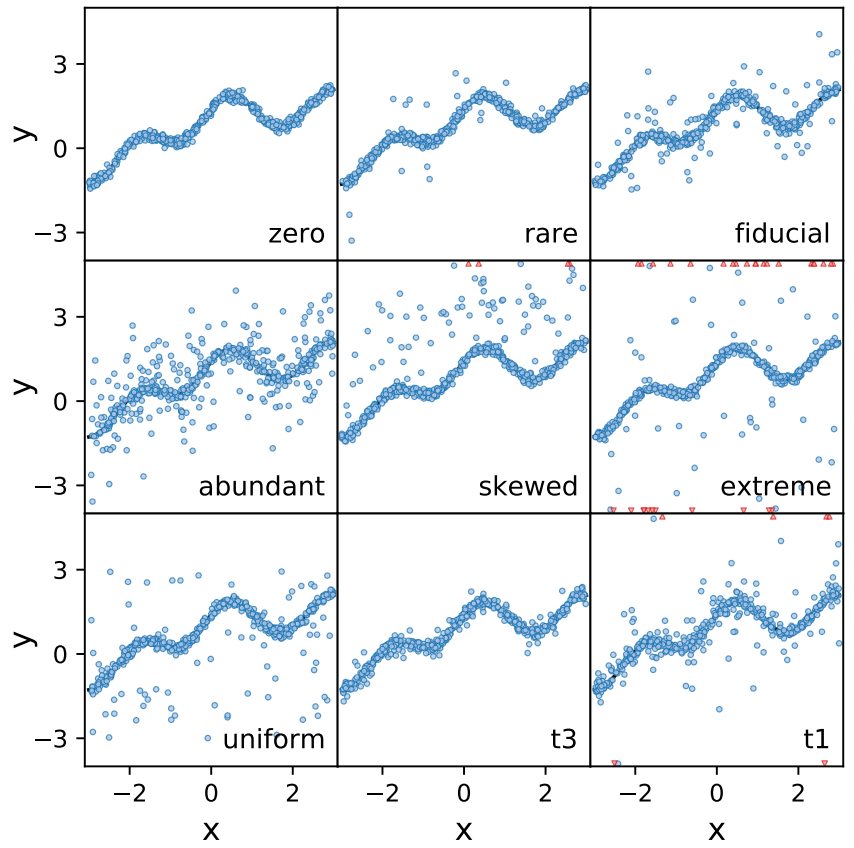

Figure 3: Example datasets for Cases 1-9. Some cases contain extreme outliers; those data points outside the box are indicated by triangles.

(see Fig. 3 for examples). We also generate larger samples with $n=500$ for comparison. A noise-free test sample of $m=2000$ points is uniformly drawn in the same range.

We adopt the following kernel function for all tested methods,

$$
k\left(x_{i}, x_{j}\right)=\sigma_{\mathrm{k}}^{2} \exp \left(-d_{i j}^{2} / 2\right)+\sigma_{\mathrm{w}}^{2} \delta_{i j},
$$

where $d_{i j}=\left|x_{i}-x_{j}\right| / l_{\mathrm{k}}$. The first term is a squared-exponential kernel, which reflects the correlation between different locations. The second term is a white noise kernel, which captures the random errors due to observation noise. In practice, a nonzero white noise term is generally recommended (even for $t$-lik $\mathrm{GP})$ to ensure valid matrix inversions. The $t$-lik GP involves two more parameters, the degree of freedom (DoF), $v$, and the scale, $\sigma_{t}$, of the Student- $t$ observation model. For each method, the parameters $\Theta=\left\{l_{\mathrm{k}}, \sigma_{\mathrm{k}}, \sigma_{w},\left[v, \sigma_{t}\right]\right\}$ are determined by maximizing the posterior probability of the training set. Specifically, we update $\Theta$ in each iteration step of ITGP.

Fig. 1 shows model predictions for one example training set, and Fig. 4 presents box plots of RMSE (see also Table 1). For Cases 8 and 9, we also show the $t$-lik GP results with DoF fixed to the true value ( 3 and 1 ) for reference (labeled as " $t$-lik GP $(v)$ ").

The standard GP works the best when the main sample is Gaussian and free of contamination (Case "zero") and still provides good results with slight non-normality (Case " $t_{3}$ "). However, it is highly susceptible to outliers in the other cases, where $t$-lik GP and ITGP show significantly better performance. $t$ lik GP seems comparable to or slightly better than ITGP for low outlier fraction cases (Case "zero", "rare", and " $t_{3}$ ") when the sample size is small $(n=100)$. In other cases, ITGP significantly outperforms $t$-lik GP, and more so for samples with $n=500$. 

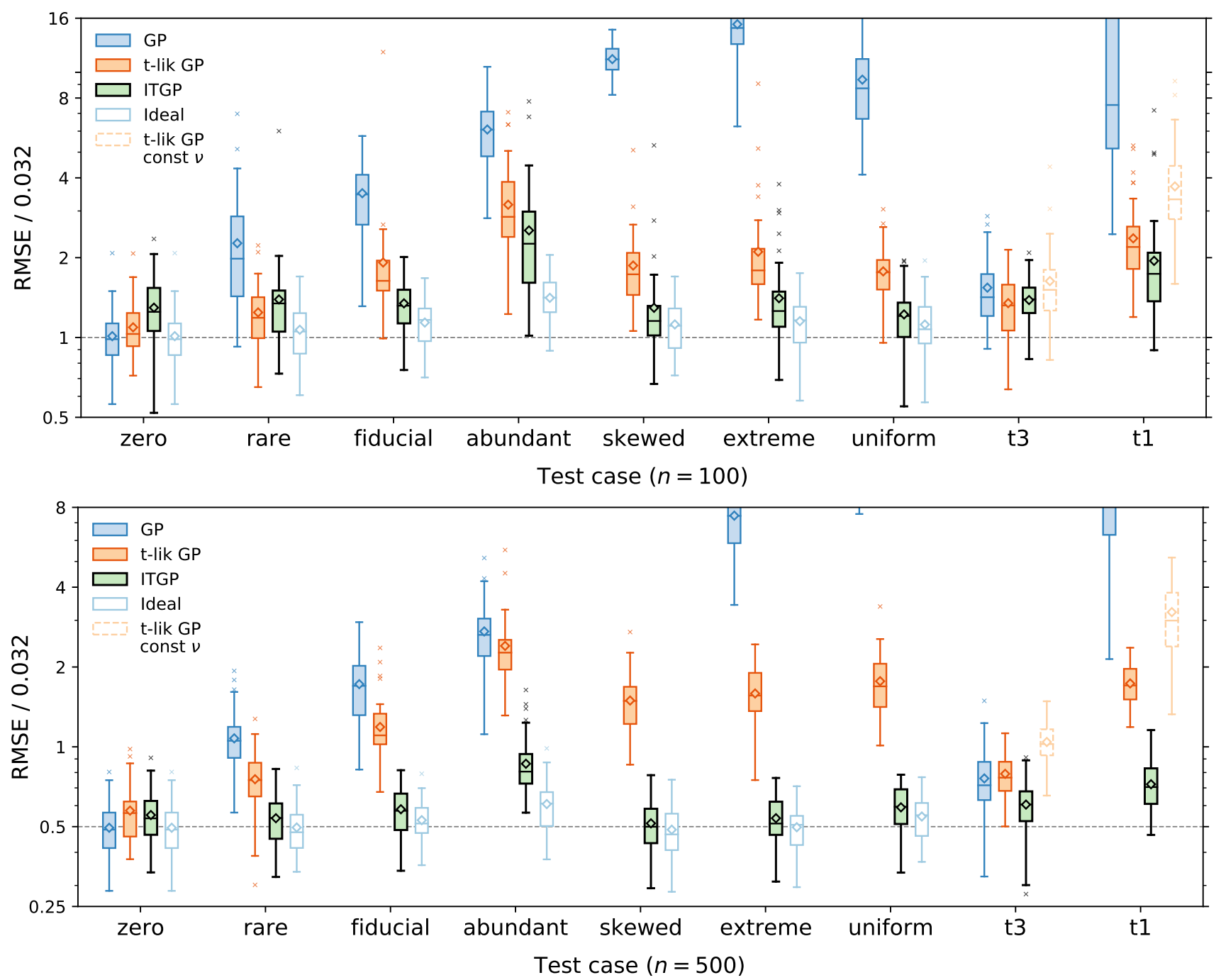

Figure 4: Performance comparison on Neal datasets. The root-mean-square errors (RMSE) are shown as box plots. In each box, the edges indicate the interquartile range, and the central line and the diamond symbol indicate the median and average, respectively.
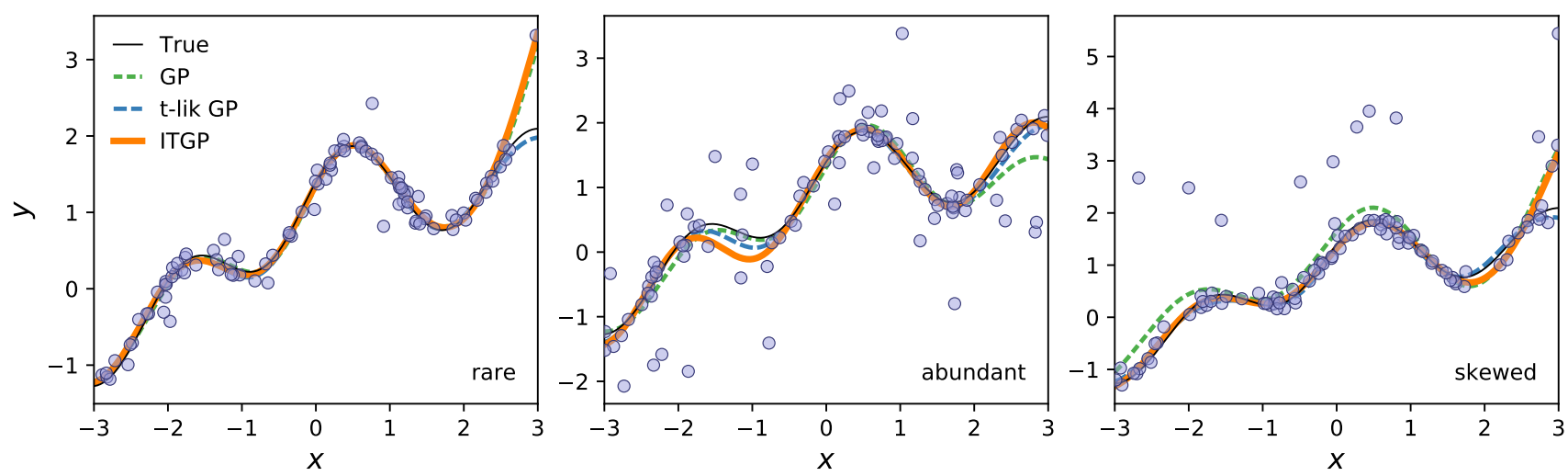

Figure 5: Three example datasets (with $n=100$ ) on which ITGP shows inferior performance. GP-based models can be biased in regions (mostly near the boundaries) where the local signal-to-noise ratio is low. This effect is less severe for larger data samples (e.g., $n=500)$.

Remarkably, in several cases, e.g., "skewed", "extreme", and "uniform", the median RMSE of ITGP even approaches the ideal performance using purified samples ("Ideal GP"). It is because outliers in these cases are more broadly dispersed and distinct from the main sample (see Fig. 3), hence in favor of trimming algorithms. Moreover, ITGP's performance is better than or at least comparable to $t$-lik GP's even in the Student- $t$ noise cases (" $t_{3}$ " and " $t_{1}$ "). It is perhaps because the long tail of Student $-t$ 
distribution leads to lower efficiency, especially when $v$ is small. We also find that when fixing $v$, even to the true value of the underlying sample, $t$-lik GP usually makes worse predictions due to less freedom in model fitting.

The RMSE of ITGP approximately reduces as $1 / \sqrt{n}$, while it does not apply to GP or $t$-lik GP in most cases. For example, in Case "skewed", the mean RMSE of GP and $t$-lik GP only reduces by $10 \%$ and $20 \%$, respectively, when the sample size increases from 100 to 500. The stars clusters in Section 3.2 make an even more extreme example. It is because the estimate's variance shrinks with larger $n$, but the bias does not. This fact implies that ITGP has a much smaller bias than GP or $t$-lik GP for contaminated samples.

In Fig. 4, several datasets show much larger RMSE than others, hence lying above the whiskers (third quartile plus 1.5 times interquartile range). As demonstrated by examples in Fig. 5, such inferior performance is mainly caused by low local signalto-noise ratio. Unlike linear regressions where data points are expected to follow a global pattern, nonlinear regressions with highly flexible models such as GP (and its variants) are more dependent on local data quality. When the local outlier fraction is high due to heavy contamination or Poisson fluctuation, outliers may have a chance to dominate the local model prediction. This effect is more severe near the boundaries and for small samples. In fact, if we leave aside the boundary regions, the average RMSE within [-2,2] of these datasets is much smaller and indistinguishable from the others. We also find that using more shrinking steps, e.g., $n_{\mathrm{sh}}=5$ or 10 rather than 2 , may help alleviate this effect in certain cases (see Fig. 8b).

Finally, we provide a comparison of computation time in Table 1 . The time cost of ITGP is typically 3.5 to 5 times that of the standard GP (see Section 2.4 for more discussion). The time cost of $t$-lik GP is significantly longer than GP, in consistency with earlier benchmarks (Jylänki et al., 2011; Ranjan et al., 2016). We avoid over-interpreting the comparison because the running time strongly depends on the implementation (e.g., inference approximation, optimizer, programming language, and parallelization $)^{5}$. Nevertheless, the relative time between the standard GP and ITGP is always meaningful.

\subsection{Star clusters}

The stars in an open cluster are believed to share the same age, chemical composition, and many other physical properties. Ideally, low-mass stars are expected to be located on a curve, the so-called main sequence, in the color-magnitude diagram (aka Hertzsprung-Russell diagram). In observation, cluster stars actually follow an extended distribution peaked around the main sequence, as shown in Fig. 6. This extension is caused by observational errors and intrinsic scatters. Moreover, the unresolved binary stars are spread broadly on the redder and brighter (righttop) side of the sequence, serving as outliers in this problem. A precise empirical determination of the main-sequence ridge line

\footnotetext{
${ }^{5}$ However, note that other $t$-lik GP implementations, e.g., the variational approximation and the scale-mixture representation with Markov chain Monte Carlo, are much slower than the Laplace approximation adopted in this test.
}

Table 1: Comparison of different models for Neal datasets.

\begin{tabular}{|c|c|c|c|c|}
\hline \multirow[t]{2}{*}{ Sample size } & \multicolumn{2}{|c|}{$n=100$} & \multicolumn{2}{|c|}{$n=500$} \\
\hline & $\frac{\text { RMSE }}{0.032}$ & Time/s & $\frac{\text { RMSE }}{0.032}$ & Time/s \\
\hline \multicolumn{5}{|l|}{ Case 1: zero } \\
\hline GP & 1.0 & 0.069 & 0.50 & 0.37 \\
\hline$t$-lik GP & 1.1 & 4.9 & 0.57 & 18 \\
\hline ITGP & 1.3 & 0.35 & 0.55 & 2.0 \\
\hline Ideal & 1.0 & 0.070 & 0.50 & 0.37 \\
\hline \multicolumn{5}{|l|}{ Case 2: rare } \\
\hline GP & 2.3 & 0.073 & 1.1 & 0.44 \\
\hline$t$-lik GP & 1.2 & 4.6 & 0.75 & 19 \\
\hline ITGP & 1.4 & 0.36 & 0.54 & 2.0 \\
\hline Ideal & 1.1 & 0.066 & 0.50 & 0.39 \\
\hline \multicolumn{5}{|l|}{ Case 3: fiducial } \\
\hline GP & 3.5 & 0.079 & 1.7 & 0.48 \\
\hline$t$-lik GP & 1.9 & 4.5 & 1.2 & 20 \\
\hline ITGP & 1.3 & 0.35 & 0.58 & 2.1 \\
\hline Ideal & 1.1 & 0.057 & 0.53 & 0.39 \\
\hline \multicolumn{5}{|l|}{ Case 4: abundant } \\
\hline GP & 6.1 & 0.085 & 2.7 & 0.51 \\
\hline$t$-lik GP & 3.2 & 2.2 & 2.4 & 12 \\
\hline ITGP & 2.5 & 0.36 & 0.86 & 2.0 \\
\hline Ideal & 1.4 & 0.033 & 0.61 & 0.28 \\
\hline \multicolumn{5}{|l|}{ Case 5: skewed } \\
\hline GP & 11 & 0.088 & 9.9 & 0.56 \\
\hline$t$-lik GP & 1.9 & 4.9 & 1.5 & 20 \\
\hline ITGP & 1.3 & 0.35 & 0.51 & 2.1 \\
\hline Ideal & 1.1 & 0.058 & 0.49 & 0.39 \\
\hline \multicolumn{5}{|l|}{ Case 6: extreme } \\
\hline GP & 15 & 0.11 & 7.4 & 0.62 \\
\hline$t$-lik GP & 2.1 & 3.5 & 1.6 & 18 \\
\hline ITGP & 1.4 & 0.38 & 0.54 & 2.2 \\
\hline Ideal & 1.2 & 0.058 & 0.50 & 0.39 \\
\hline \multicolumn{5}{|l|}{ Case 7: uniform } \\
\hline GP & 15 & 0.10 & 12 & 0.56 \\
\hline$t$-lik GP & 2.6 & 3.0 & 1.8 & 19 \\
\hline ITGP & 1.6 & 0.37 & 0.59 & 2.1 \\
\hline Ideal & 1.3 & 0.046 & 0.55 & 0.33 \\
\hline \multicolumn{5}{|l|}{ Case 8: $t_{3}$} \\
\hline GP & 1.5 & 0.071 & 0.76 & 0.42 \\
\hline$t$-lik GP & 1.3 & 3.6 & 0.79 & 15 \\
\hline ITGP & 1.4 & 0.35 & 0.61 & 1.9 \\
\hline$t$-lik GP $(v=3)$ & 1.6 & 4.3 & 1.0 & 20 \\
\hline \multicolumn{5}{|l|}{ Case 9: $t_{1}$} \\
\hline GP & 110 & 0.10 & 79 & 0.76 \\
\hline$t$-lik GP & 2.4 & 3.2 & 1.7 & 15 \\
\hline ITGP & 1.9 & 0.37 & 0.72 & 2.3 \\
\hline$t$-lik GP $(v=1)$ & 3.7 & 3.5 & 3.2 & 16 \\
\hline
\end{tabular}

The smallest RMSE for each dataset is marked in bold. 

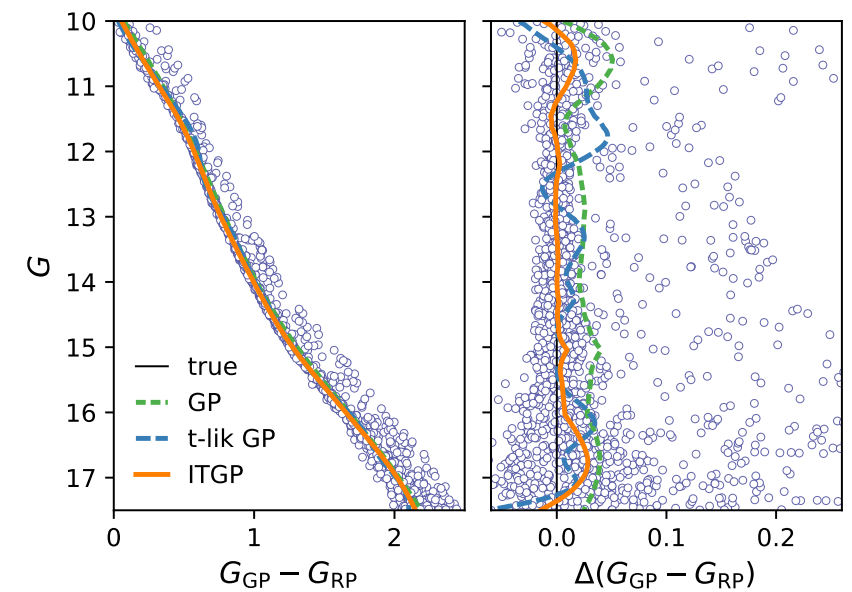

Figure 6: Color-magnitude diagram of a synthetic open cluster with 500 stars. Most single stars are distributed around the main-sequence ridge line, while the binary stars are extensively spread towards the upper right (more luminous and redder) side. The right panel shows the residual colors from the true ridge line. The curves present the model color, $G_{\mathrm{GP}}-G_{\mathrm{RP}}$, as a function of $G$ band magnitude predicted by three GP methods, respectively.

in observation is crucial to many studies, including inferring binary star properties and calibrating theoretical stellar models (see Li et al. 2020). As demonstrated by Li et al. (2020), the proposed ITGP can pinpoint the ridge line with high precision.

In the following, we present a benchmark test using the realistic synthetic clusters generated by Li et al. (2020). Assuming a stellar mass function and a binary mass-ratio distribution, $2 \times 50$ open clusters are generated with the PARSEC stellar model (Bressan et al., 2012), each containing 500 or 1000 stars, $\sim 30 \%$ of which are binary stars (outliers). To mimic the observation from the Gaia mission, we add a magnitude-dependent noise on each star, making it a heteroscedastic problem. We aim to predict the color, $G_{\mathrm{BP}}-G_{\mathrm{RP}}$, as a function of the magnitude, $G$, along the ridge line of the main sequence (see Fig. 6).

We first adopt the squared-exponential kernel (Equation 6) as the previous subsection. Stein (1999) argues that the weaker smoothness of the Matérn kernels makes them more realistic for modeling many physical processes. Therefore, we also try the following kernels separately: a composite Matérn 5/2 kernel,

$$
k\left(x_{i}, x_{j}\right)=\sigma_{\mathrm{k}}^{2}\left(1+\sqrt{5} r_{i j}+5 r_{i j} / 3\right) \exp \left(-\sqrt{5} r_{i j}\right)+\sigma_{\mathrm{w}}^{2} \delta_{i j},
$$

and a composite Matérn 3/2 kernel,

$$
k\left(x_{i}, x_{j}\right)=\sigma_{\mathrm{k}}^{2}\left(1+\sqrt{3} r_{i j}\right) \exp \left(-\sqrt{3} r_{i j}\right)+\sigma_{\mathrm{w}}^{2} \delta_{i j},
$$

where $r_{i j}=\left|x_{i}-x_{j}\right| / l_{\mathrm{k}}$. Same as the Neal benchmarks, the parameters, $\Theta=\left\{l_{\mathrm{k}}, \sigma_{\mathrm{k}}, \sigma_{w},\left[v, \sigma_{t}\right]\right\}$ are determined by maximizing the posterior probability of the training sample.

Fig. 6 shows model predictions using the Matérn 5/2 kernel for one of the synthetic clusters, while Fig. 7 presents the box plot for the overall performance with different kernels (see also Table 2). In general, the results are not sensitive to the choice of kernel, though the Matérn 5/2 kernel seems slightly favored by t-lik GP. The proposed ITGP again shows significantly better

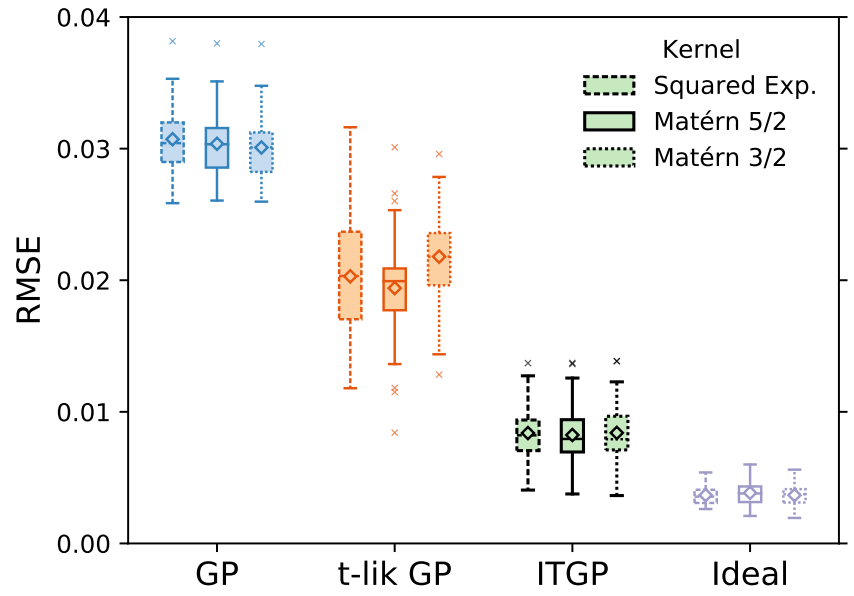

Figure 7: Performance comparison on synthetic star clusters. Each cluster contains 1000 stars.

Table 2: Comparison of different models for star cluster datasets.

\begin{tabular}{lcccc}
\hline Sample size & \multicolumn{2}{c}{$n=500$} & \multicolumn{2}{c}{$n=1000$} \\
\hline & RMSE & Time/s & RMSE & Time/s \\
\hline GP & & & & \\
Squared Exp & 0.031 & 0.51 & 0.031 & 2.2 \\
Matérn 5/2 & 0.031 & 0.61 & 0.030 & 2.2 \\
Matérn 3/2 & 0.031 & 0.81 & 0.030 & 2.9 \\
-lik GP & & & & \\
Squared Exp & 0.020 & 15 & 0.020 & 55 \\
Matérn 5/2 & 0.020 & 17 & 0.019 & 58 \\
Matérn 3/2 & 0.021 & 21 & 0.022 & 54 \\
ITGP & & & & \\
Squared Exp & 0.0094 & 2.3 & 0.0084 & 6.4 \\
Matérn 5/2 & $\mathbf{0 . 0 0 9 2}$ & 2.8 & $\mathbf{0 . 0 0 8 2}$ & 7.4 \\
Matérn 3/2 & 0.0092 & 3.8 & 0.0084 & 10 \\
Ideal & & & & \\
Squared Exp & 0.0064 & 0.45 & 0.0037 & 0.89 \\
Matérn 5/2 & 0.0060 & 0.48 & 0.0038 & 1.1 \\
Matérn 3/2 & 0.0052 & 0.68 & 0.0037 & 1.5 \\
\hline
\end{tabular}

performance than GP and $t$-lik GP in this problem, where the data is contaminated by abundant and skewed outliers (binary stars).

It is noteworthy that ITGP with a simple homoscedastic kernel provides remarkable performance even in this heteroscedastic problem. Nevertheless, using a universal variance will trim more data points than optimal in the region where the noise is greater than average (e.g., at $G \simeq 17$ ), which may lower the local efficiency and lead to bias. It is probably why doubling the sample size only brings minor improvement in the RMSE of ITGP. The results can be further improved by using heteroscedastic kernels in principle. We leave such exploration to future work.

\section{Conclusion}

This work has proposed a new robust regression algorithm based on the Gaussian process and iterative trimming (ITGP). 
It greatly improves the model accuracy of GP in the presence of outliers by iteratively removing the most extreme data points. A novel shrinking procedure that gradually increases the trimming fraction is introduced to prevent premature convergence, and a one-step reweighting is used to increase the statistical efficiency. The advantage of ITGP lies in its robustness, efficiency, ease of implementation, and computational tractability.

Applied to a wide range of synthetic datasets with different contamination levels, ITGP significantly outperforms the standard GP and the popular robust GP variant with the Student- $t$ likelihood in most test cases, including some particularly challenging problems with excessive contamination fraction (45\%), extremely distributed outliers, or moderate heteroskedasticity.

Though the optimal method always depends on the specific problem in principle, ITGP, nevertheless, shows remarkable performance as a general method, thus ensuring wide application. In addition, ITGP may serve as a good initial estimate for other advanced robust estimators (e.g., Yohai 1987; Gervini and Yohai 2002) for possible future improvement.

We have made our implementation of ITGP available online (https://github.com/syrte/robustgp/), which is written in Python based on the public GP package GPY. It is also straightforward to implement ITGP with other GP packages for better speed and scalability.

\section{Acknowledgments}

We thank Jiaxin Han and Hai Yu for helpful discussions and the anonymous referees for constructive criticisms and suggestions. This work is supported by National Key Basic R\&D Program of China (2018YFA0404504, 2019YFA0405501), NSFC (11621303, 11873038, 11890691, 11973032, 12022307, U2031139), and 111 project (B20019). ZZL gratefully acknowledges the support of the MOE Key Lab for Particle Physics, Astrophysics and Cosmology, Ministry of Education. The computation of this work is partly done on the GRAVITY supercomputer at the Department of Astronomy, Shanghai Jiao Tong University.

During the preparation of this work, we made use of the following open source software: GPML ${ }^{6}, \mathrm{GPy}^{7}$, Jupyter ${ }^{8}$, Matplotlib $^{9}$, Numpy ${ }^{10},{\text { Oct } 2 \mathrm{Py}^{11} \text {, Octave }}^{12}$, Python ${ }^{13}$, and Scipy ${ }^{14}$.

\section{Appendix A. Choice of ITGP hyperparameters}

This appendix presents a series of numerical experiments aiming to understand the ITGP hyperparameters and find the possible optimal choice. We consider the following parameters: the trimming and reweighting parameters, $\alpha_{1}$ and $\alpha_{2}$, iteration

\footnotetext{
${ }^{6}$ http://www.gaussianprocess.org/gpml/code/

${ }^{7}$ https://github.com/SheffieldML/GPy

${ }^{8}$ https://jupyter.org/

${ }^{9}$ https://matplotlib.org/

${ }^{10}$ https://numpy.org/

${ }^{11}$ https://github.com/blink1073/oct2py

${ }^{12}$ https://www.gnu.org/software/octave/

${ }^{13}$ https://www . python.org/

${ }^{14}$ https://www. scipy.org/
}

numbers for shrinking, concentrating, and reweighting stages, $n_{\mathrm{sh}}, n_{\mathrm{cc}}$, and $n_{\mathrm{rw}}$. We systematically change one or several of the above parameters and perform the same tests on the synthetic Neal datasets introduced in Section 3.1. The results with sample size $n=100$ are shown in Fig. 8. We do not find any significant dependence of the following conclusions on the sample size.

In general, we find the fiducial values used in the main text, $\alpha_{1}=0.5, \alpha_{2}=0.975, n_{\mathrm{sh}}=3, n_{\mathrm{cc}}=2$, and $n_{\mathrm{rw}}=1$ (one-step reweighting), work remarkably well for all test cases. Although the best configuration can depend on the specific problem, the improvement from further tuning is usually limited. Below we summarize some general expectations about hyperparameters and performance.

- $\alpha_{1}$. Using a larger $\alpha_{1}$, e.g., 0.75 , can mildly increase the

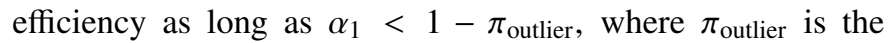
outlier fraction (Fig. 8a). However, if $\alpha_{1}$ is too large, e.g., 0.75 for Case "abundant" and "uniform" $\left(\pi_{\text {outlier }}=0.45\right.$ and 0.3$)$, the performance may get significantly worsen due to the incomplete exclusion of influential outliers. Therefore, 0.5 is recommended for maximum robustness, unless one knows the upper limit of the outlier fraction in prior.

- $n_{\text {sh. }}$. The shrinking steps can effectively prevent premature convergence (Fig. 2). While the average performance is not very sensitive to $n_{\text {sh }}$ once $n_{\text {sh }} \geq 2$ (Fig. $8 \mathrm{~b}$ ), a larger $n_{\text {sh might help }}$ improve the inferior performance on some particular datasets (e.g., for Case "extreme").

- $n_{\mathrm{cc}}$. The number of concentrating steps $n_{\mathrm{cc}}$ has little effect on ITGP performance in most cases (Fig. 8c). The concentrating stage can approach or even achieve convergence within two or three steps (see also Fig. 2), though several more steps are generally needed for exact convergence when the sample is large. Comparing with $n_{\mathrm{cc}}=2$, we find that allowing complete convergence makes little difference, especially when a successive reweighting is added. Larger $n_{\mathrm{cc}}$ is slightly favored when $\alpha_{1}$ is close to $1-\pi_{\text {outlier }}$ (e.g., in Case "abundant"). A rule of thumb is to ensure at least 2 or 3 iterations (including shrinking steps) with $\alpha<1-\pi_{\text {outlier }}$ before the final reweighting.

- $\alpha_{2}$. Using $\alpha_{2}=0.95,0.975$, or 0.997 typically gives very similar results, though one of them can be slightly favored depending on the nature of outliers (Fig. 8a). Using even larger values is not recommended. For an obvious example, it turns back to the standard GP with $\alpha_{2}=1$.

- $n_{\mathrm{rw}}$. The one-step reweighting $\left(n_{\mathrm{rw}}=1\right)$ can significantly improve the model precision if $\alpha_{1} \ll 1-\pi_{\text {outlier }}$ but slightly worsen the result otherwise (Fig. 8d). It is because the efficiency after the concentrating stage is already close to optimal in the latter case (e.g., Case "abundant"). One may wonder what if performing iterative reweighting steps in a way similar to concentrating steps. We find that using $n_{\mathrm{rw}}>1$ can slightly reduce RMSE in several cases at the cost of more computation (Fig. 8d). Interestingly, a naive approach (labeled as "rw only") using iterative reweighting without preceding shrinking and concentrating steps (adopted by e.g., Wang et al. 2017) also shows comparable performance in several cases. However, this variant is significantly less favorable for heavily contaminated cases ("abundant" and "uniform") compared with the proposed ITGP. 

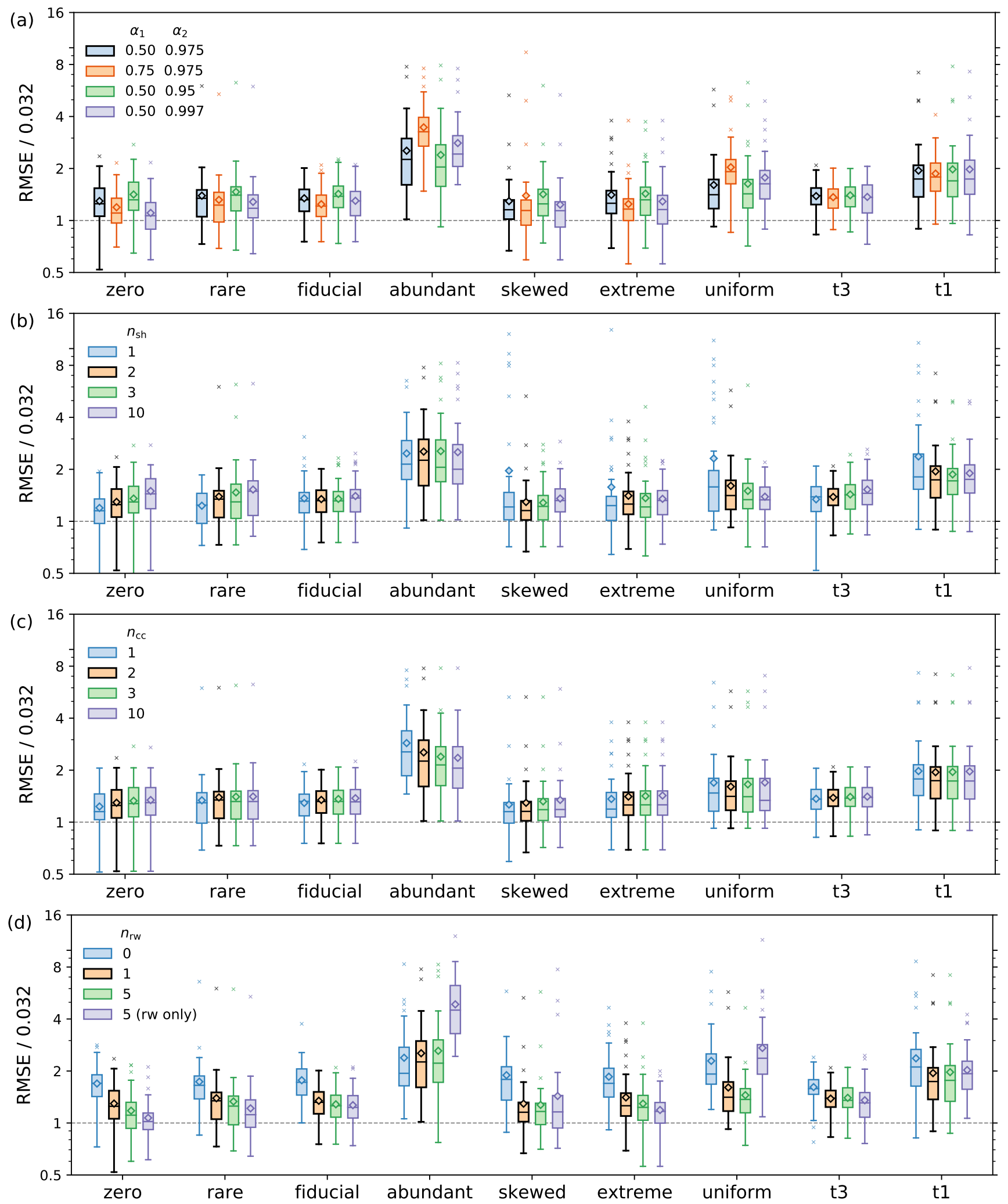

Figure 8: Similar to Fig. 4, but showing the dependence of ITGP's performance on hyperparameters for test cases with $n=100$. In each panel, one or several parameters have been changed from the fiducial values. The fiducial configuration $\left(\alpha_{1}=0.5, \alpha_{2}=0.975, n_{\mathrm{sh}}=3, n_{\mathrm{cc}}=1\right.$, and $\left.n_{\mathrm{rw}}=1\right)$ is marked by a black box in each test case. 
In summary, our recommended parameters seem a good compromise among robustness, efficiency, and computation cost for general problems.

\section{References}

Almosallam, I., 2017. Heteroscedastic Gaussian Processes for Uncertain and Incomplete Data. Ph.D.. University of Oxford.

Ambikasaran, S., Foreman-Mackey, D., Greengard, L., Hogg, D.W., O’Neil, M., 2015. Fast Direct Methods for Gaussian Processes. IEEE Transactions on Pattern Analysis and Machine Intelligence 38, 252. doi:10 . 1109/TPAMI. 2015.2448083, arXiv: 1403.6015.

Bressan, A., Marigo, P., Girardi, L., Salasnich, B., Dal Cero, C., Rubele, S., Nanni, A., 2012. PARSEC: stellar tracks and isochrones with the PAdova and TRieste Stellar Evolution Code. Monthly Notices of the Royal Astronomical Society 427, 127-145. doi:10.1111/j.1365-2966.2012.21948.x, arXiv: 1208.4498 .

Croux, C., Haesbroeck, G., 1999. Influence Function and Efficiency of the Minimum Covariance Determinant Scatter Matrix Estimator. Journal of Multivariate Analysis 71, 161-190. doi:10.1006/jmva. 1999.1839.

Duvenaud, D., 2014. Automatic Model Construction with Gaussian Processes. Thesis. University of Cambridge. doi:10.17863/CAM. 14087.

Foreman-Mackey, D., Agol, E., Ambikasaran, S., Angus, R., 2017. Fast and Scalable Gaussian Process Modeling with Applications to Astronomical Time Series. Astronomical Journal 154, 220. doi:10.3847/1538-3881/ aa9332, arXiv: 1703.09710 .

Gervini, D., Yohai, V.J., 2002. A class of robust and fully efficient regression estimators. Annals of Statistics 30, 583-616. doi:10. 1214/aos/1021379866.

Goldberg, P.W., Williams, C.K.I., Bishop, C.M., 1998. Regression with inputdependent noise: A Gaussian process treatment, in: Proceedings of the 1997 Conference on Advances in Neural Information Processing Systems 10, MIT Press, Cambridge, MA, USA. pp. 493-499.

Heitmann, K., Higdon, D., White, M., Habib, S., Williams, B.J., Lawrence, E., Wagner, C., 2009. The Coyote Universe. II. Cosmological Models and Precision Emulation of the Nonlinear Matter Power Spectrum. Astrophysical Journal 705, 156-174. doi:10. 1088/0004-637X/705/1/156, arXiv:0902 .0429.

Hensman, J., Fusi, N., Lawrence, N.D., 2013. Gaussian processes for Big data, in: Proceedings of the Twenty-Ninth Conference on Uncertainty in Artificial Intelligence, AUAI Press, Bellevue, WA. pp. 282-290.

Huber, P.J., 1964. Robust Estimation of a Location Parameter. The Annals of Mathematical Statistics 35, 73-101. doi:10.1214/aoms/1177703732.

Huber, P.J., Ronchetti, E.M., 2009. Robust Statistics. John Wiley \& Sons, Inc. doi:10.1002/9780470434697.

Jylänki, P., Vanhatalo, J., Vehtari, A., 2011. Robust Gaussian Process Regression with a Student-t Likelihood. Journal of Machine Learning Research 12, 3227-3257.

Kuß, M., 2006. Gaussian Process Models for Robust Regression, Classification, and Reinforcement Learning. Ph.D. thesis. Technische Universität. Darmstadt.

Li, L., Shao, Z., Li, Z.Z., Yu, J., Zhong, J., Chen, L., 2020. Modeling Unresolved Binaries of Open Clusters in the Color-Magnitude Diagram. I. Method and Application of NGC 3532. Astrophysical Journal 901, 49. doi:10.3847/ 1538-4357/abaef3, arXiv:2008.04684.

Liu, H., Ong, Y.S., Shen, X., Cai, J., 2020. When Gaussian Process Meets Big Data: A Review of Scalable GPs. IEEE Transactions on Neural Networks and Learning Systems 31, 4405-4423. doi:10.1109/TNNLS. 2019.2957109.
Maronna, R.A., Martin, R.D., Yohai, V.J., Salibián-Barrera, M., 2019. Robust Statistics: Theory and Methods (with R). John Wiley \& Sons.

Martinez-Cantin, R., Tee, K., McCourt, M., 2018. Practical Bayesian optimization in the presence of outliers, in: International Conference on Artificial Intelligence and Statistics, PMLR. pp. 1722-1731.

McClintock, T., Rozo, E., Becker, M.R., DeRose, J., Mao, Y.Y., McLaughlin, S., Tinker, J.L., Wechsler, R.H., Zhai, Z., 2019. The Aemulus Project. II. Emulating the Halo Mass Function. Astrophysical Journal 872, 53. doi:10. 3847/1538-4357/aaf568, arXiv: 1804.05866.

Naish-Guzman, A., Holden, S., 2007. Robust regression with twinned Gaussian processes, in: Proceedings of the 20th International Conference on Neural Information Processing Systems, Curran Associates Inc., Red Hook, NY, USA. pp. 1065-1072.

Neal, R.M., 1997. Monte Carlo Implementation of Gaussian Process Models for Bayesian Regression and Classification. arXiv e-prints , physics/9701026arXiv: physics/9701026.

Pison, G., Van Aelst, S., Willems, G., 2002. Small sample corrections for LTS and MCD. Metrika 55, 111-123. doi:10.1007/s001840200191.

Ramirez-Padron, R., Mederos, B., Gonzalez, A.J., 2021. Robust weighted Gaussian processes. Computational Statistics 36, 347-373. doi:10.1007/ s00180-020-01011-0.

Ranjan, R., Huang, B., Fatehi, A., 2016. Robust Gaussian process modeling using EM algorithm. Journal of Process Control 42, 125-136. doi:10. 1016/ j.jprocont. 2016.04 .003 .

Rasmussen, C.E., Williams, C.K.I., 2005. Gaussian Processes for Machine Learning. The MIT Press. doi:10.7551/mitpress/3206.001.0001.

Ross, J.C., Dy, J.G., 2013. Nonparametric mixture of Gaussian processes with constraints, in: Proceedings of the 30th International Conference on International Conference on Machine Learning - Volume 28, JMLR.org, Atlanta, GA, USA. pp. III-1346-III-1354.

Rousseeuw, P.J., 1984. Least Median of Squares Regression. Journal of the American Statistical Association 79, 871-880. doi:10.1080/01621459. 1984. 10477105 .

Rousseeuw, P.J., Driessen, K., 2006. Computing LTS Regression for Large Data Sets. Data Mining and Knowledge Discovery 12, 29-45. doi:10. $1007 / \mathrm{s} 10618-005-0024-4$.

Rousseeuw, P.J., Leroy, A.M., 1987. Robust Regression and Outlier Detection. John Wiley \& Sons, Inc., New York, NY, USA.

Stegle, O., Fallert, S.V., MacKay, D.J.C., Brage, S., 2008. Gaussian Process Robust Regression for Noisy Heart Rate Data. IEEE Transactions on Biomedical Engineering 55, 2143-2151. doi:10.1109/TBME. 2008. 923118.

Stein, M.L., 1999. Interpolation of Spatial Data: Some Theory for Kriging. Springer Series in Statistics, Springer-Verlag, New York. doi:10.1007/ 978-1-4612-1494-6.

Vanhatalo, J., Jylänki, P., Vehtari, A., 2009. Gaussian process regression with Student-t likelihood, in: Bengio, Y., Schuurmans, D., Lafferty, J.D., Williams, C.K.I., Culotta, A. (Eds.), Advances in Neural Information Processing Systems 22. Curran Associates, Inc., pp. 1910-1918.

Wang, D., Xue, J., Cui, D., Zhong, Y., 2017. A robust submap-based road shape estimation via iterative Gaussian process regression, in: 2017 IEEE Intelligent Vehicles Symposium (IV), IEEE, Los Angeles, CA, USA. pp. 1776-1781. doi:10.1109/IVS.2017.7995964.

Yohai, V.J., 1987. High Breakdown-Point and High Efficiency Robust Estimates for Regression. The Annals of Statistics 15, 642-656. doi:10.1214/aos/ 1176350366.

Yu, C., Yao, W., 2017. Robust linear regression: A review and comparison. Communications in Statistics - Simulation and Computation 46, 6261-6282. doi:10.1080/03610918.2016.1202271. 